\title{
Positive Approaches to Care: a new look at dementia education
}

Murphy J (20I7) Positive Approaches to Care: a new look at dementia education. Primary Health Care. 27, I, $29-33$.

Date of submission: 12 April 2016; Date of acceptance: 27 June 2016. doi: 10.7748/phc.2017.ell57

Jayne Murphy

Senior lecturer, Institute

of Health Professions,

University of Wolverhampton,

Wolverhampton

\section{Correspondence}

j.s.murphy@wlv.ac.uk

\section{Conflict of interest}

None declared

\section{Peer review}

This article has been subject to external double-blind peer review and checked for plagiarism using automated software

\begin{abstract}
This article provides an overview of relevant factors in dementia care. The number of people experiencing dementia is increasing and dementia is at the forefront of health policy. The evidence base for personcentred approaches and interventions in dementia is expanding and nurses are central to implementing these across care settings. This is a challenging time for dementia care and nursing has a major role in leading and developing these changes in practice. The article discusses the Positive Approaches to Care (PAC) philosophy as a training approach in caring for people with dementia.
\end{abstract}

\section{Keywords}

Alzheimer's, community nursing, dementia, dementia education, dementia training, positive approach to care, primary care

THERE ARE 850,000 people in the UK with dementia and this figure is set to rise to more than 2 million by 2051 (Alzheimer's Society 2014). Dementia is not a diagnosis but a term used to describe the symptoms of a condition, illness or syndrome which results in physical damage to areas of the brain. It is usually progressive and irreversible, and characterised by symptoms of deteriorating cognition, such as impaired memory, co-ordination and judgment (Whalley and Breitner 2009). It is assumed that over time, someone who has dementia will depend more on others for all aspects of care (O'Connor and Purves 2009).

There is now far greater knowledge about the causes of dementia and the abilities that remain despite the loss of cognition. This poses a challenge to past ideas of dementia, and raises questions about how people with dementia are regarded and respected (Nuffield Council on Bioethics 2009).

Dementia is a growing concern internationally; the number of people developing dementia is increasing and this has associated financial and personal consequences. The focus is on improving all aspects of dementia care, from research to the education and training of professional and informal carers, to reduce the effect of dementia on individuals and society.

Another priority is improving and sustaining the quality of life of those affected by dementia, including carers. This article highlights a training approach from the US that can support community and primary care nurses to provide better dementia care.

\section{Dementia resources}

For healthcare professionals with an interest in dementia care and dementia training, search engines, social networking sites, blogs and video-sharing sites offer a multitude of resources from around the world that can prove invaluable in the learning process. Although some are dubious in content, many are reliable, informative and signify a passion for dementia care.

The Prime Minister's Challenge on Dementia 2020 (Department of Health 2015) discusses the desire for the UK to be a world leader in many areas of dementia care and research. It highlights where the UK is ahead and setting the standards for dementia care. The US, Canada and Australia are competing for the title of world leader, however, and have some excellent examples of good practice that the UK could adopt. International dementia experts, including 'expert patients' (people with dementia), are collaborating to educate the public about dementia care. It is an exciting time and an opportunity for healthcare professionals to develop their knowledge and competence.

\section{Dementia training}

The National Dementia Audit (Royal College of Psychiatrists (RCP) 2013) acknowledged that dementia education and training have 
Write for us

journals.rcni.com/r/

phc-author-guidelines

\section{Online archive}

For related information, visit primaryhealthcare. com and search using the keywords become more widely available as policy commitments and organisations have increased access to training, but it wants to see further improvements. The previous audit (RCP 2011) recommended that $100 \%$ of staff be aware of dementia, $50 \%$ be knowledgeable and $10 \%$ be specialists. In its 2013 report the RCP focused only on awareness, reporting that this still required improvement, and did not refer to the other levels of training. Both audits used data from acute hospitals and not community providers.

Health Education England (HEE) (2015) discussed dementia education and training in three tiers: awareness, basic skills and leadership. Although it focused on England, HEE referenced Dementia UK (2013), which is UK-wide, and recommended levels of dementia education across higher education. Training requirements for unregistered members of the health and social care workforce were also given. NHS Education Scotland (2011) referred to different levels of dementia education: informed, skilled, enhanced and expert. The Welsh Assembly Government (2011) incorporated a dementia training initiative and the Department of Health, Social Services and Public Safety (2011) discussed the requirement for and commitment to dementia training.

These policy documents show the commitment across the UK to improving dementia education in the workforce, but they also add to the plethora of information that individuals need to navigate to become more educated in dementia and its associated issues.

A variety of training programmes is available in the UK to increase knowledge, skills, competence and confidence in caring for people with dementia. These programmes involve different approaches, including activities such as presentations, group exercises, discussion and video clips. Some education packages - for example, the Getting to Know Me training programme (Elvish et al 2014, 2016) - have been evaluated and evidence generated to demonstrate their success in increasing the knowledge and confidence of those who have attended the training.

Evaluations of training incorporating the person-centred Dementia VIPS approach and Dementia Care Mapping (Brooker 2007) have demonstrated positive effects for individuals who have attended the training (Passalacqua and Harwood 2012). Chater and Hughes (2013)'s paper on dementia education and training acknowledged that theoretical and practical skills are important for nurses providing dementia care, suggesting education improves attitudes, but also emphasising that practical skills are just as valued by clinical staff. Smythe et al (2014) discussed a brief psychosocial training intervention in an acute hospital setting and, although the results from an evaluation were statistically insignificant, qualitative feedback from staff who had undertaken the training was positive; the authors also asserted that practical, hands-on training is required in clinical settings.

Dementia education and training should include direct practical competencies required for effective care (Jones et al 2013). Professional and informal carers need practical tips on how to care appropriately for and respond sensitively and receptively to someone with dementia.

\section{Positive Approaches to Care}

'Positive Approach to Care' (PAC) offers practical approaches to dementia care (Snow 2016a). It is a philosophy incorporating education and training conceived by Teepa Snow, a US educator and occupational therapist with more than 30 years' experience of working with people who have dementia. The PAC concept uses knowledge and practical interventions to aid in the development of a partnership between the carer and the person with dementia. PAC endorses the term 'brain change' for dementia, explaining that as the structure and function of the brain alter, the person with dementia may change in response - particular symptoms may develop, abilities may change, and understanding and response may alter. There are currently 15 PAC certified trainers in the UK and Ireland (Snow 2016a).

Another 16 people started certification training in November 2016; they will be added to the approved trainers list when mentorship is completed. Training is offered throughout the UK from certified trainers at a range of organisations who are embedding the approach into their service areas. These include residential care providers such as Norse Care (www.norsecare.co.uk), higher education institutions such as the University of Wolverhampton (www.wlv.ac.uk), NHS providers, voluntary organisations and social enterprises, such as Engage and Create (www. engageandcreate.com/).

Raymond et al (2014)'s evaluation of educational initiatives in palliative care in dementia supported the use of Kolb's (1984) experiential learning theory, which relates concrete practice to conceptual models. They suggested that learning should be about enhancing performance, emphasising the resolution of clinical concerns and better outcomes for patients. Integrated learning with practice is more relevant to the learner. 
PAC training is based on the experiential learning cycle and is practical and logical. It helps to make sense of what it might be like to have dementia; how surroundings, the environment, people and care might be interpreted; and the responses these factors might evoke from all involved. For example, when someone with dementia receives an assisted wash, they might struggle to understand what is happening and may not acquiesce to it being 'done to' them. PAC helps carers to appreciate what someone with dementia may be experiencing and to adapt their approach to accommodate the individual needs of the people receiving the care.

There are three core aspects of PAC. The first is the 'gems' concept, in which people with dementia are viewed as precious gems (sapphire, diamond, emerald, amber, ruby or pearl), with the focus being on the skills that remain rather than those lost. Changes in the brain and why people with dementia may behave in a certain way are explained to carers, which supports and helps them to appreciate what it is like to have dementia, to accept that someone with dementia is unable to change and to be flexible in their approach. This makes care easier by matching the approach with the needs of the individual.

The second concept is a practical and physical method of working with the person with dementia to connect and provide care on several levels (Snow 2016a).

The third concept is connecting with the person with dementia following invitation, to enhance the communication and receptiveness of both parties.

'Gems' is Snow's creation (Snow 2016b). It evolved from the Allen cognitive levels, which measure the severity of disability in terms of the ability to process information (Maddox and Burns 1997). Snow's interpretation explains abilities and disabilities positively, using factual explanations sensitively described, which helps to promote a positive attitude to brain change. The gems are not negative labels but indicators to assess levels of care; each gem highlights remaining abilities, notes skills that are lost, and indicates how to provide better support and encouragement. The approach deliberately steers clear of stages.

For example, people with late changes at the end of their 'journey' may display characteristics of a pearl - like a pearl in an oyster shell, they are hidden inside the shells of their bodies (Box 1). Ruled primarily by reflexes, their muscles tend to be active most of the time, resulting in contractures that cannot be stopped. Although it appears they are unaware of the world around them, there will be moments when they are responsive and alert - the shell opens and the person shines through (Snow 2016b). The specific gem categories identified are (Snow 2016b):

"Sapphire - optimal cognition, healthy brain

»Diamond - clear and sharp, routines and rituals rule

»Emerald - green and on the go with a purpose, naturally flawed

"Amber - caught in a moment of time, caution required

» Ruby - deep and strong in colour, others stop seeing what is possible

" Pearl - hidden in a shell, beautiful moments to behold

PAC also connects the physical manifestations of dementia with the structural changes in the brain, supporting carers to appreciate what people who have dementia may be experiencing. For example, visual spatial impairment is often a symptom of dementia (Possin 2010), with the field of vision narrowing as the dementia progresses, affecting the ability to visually process the environment and appreciate surroundings. Someone who has dementia may therefore not be able to see a plate of food that is 'under their nose' when their head is in the natural upright position.

However, they may be able to see the plate of food opposite - that actually belongs to someone - and reach for that instead. Placing the plate in the individual's field of vision will help them to understand its location, as well as avoid accusations and the repercussions of attempting to eat someone else's food. The

\section{BOX 1. Example of PAC in practice}

Margaret has advanced Alzheimer's disease and is nursed primarily in bed. She lies with her eyes closed apparently disengaged from her surroundings. The aim in using PAC to connect and care for Margaret, is to minimise potential distress from intimate care.

Margaret would be considered 'a pearl' in PAC's gems approach. As a pearl, Margaret is nearing the end of her life, even though she may not currently be 'end of life'. Her brain is functioning at such a reduced level that she is led by reflexes; her muscles are probably 'switched on' most of the time, resulting in contractures or the inability to move independently and flexibly. It is likely that she relies on another for most of her care, most of the time. With sensitive care, Margaret may well be able to engage with her surroundings and her carers. Appreciating skills that that are retained in the right side of the brain very late on in dementia enables Margaret's carers to connect with her - by using music, rhythm, song or poetry, they can perhaps invoke interaction.

Positive physical approach guides the carer to pause at the edge of Margaret's personal space to attempt to connect with her. Introductions, saying Margaret's name and a firm but gentle touch on her ankle to let her know the carer is there, are less likely to startle her than an immediate approach and whispering in her ear - entering her intimate space without warning.

If after this Margaret is receptive to holding hands, hand-under-hand can be initiated in numerous ways, to help Margaret to take a drink, have something to eat, comb her hair or wash her face, for example. The technique helps Margaret to feel a sense of control, even this late on in the disease. 


\section{Find out more}

Visit www.teepasnow. com where you will find information and videos demonstrating the approach.
PAC approach to training helps the learner to experience this through an interactive activity, rather than just an explanation of the facts.

The focus of PAC is its practical and physical method of working with someone who has dementia. This is grounded in theory, with the notion of 'spatial behaviour' covered, encouraging the carer to appreciate boundaries and approach the person who has dementia with sensitive awareness of these boundaries.

PAC encourages carers to reflect on their regular behaviours and consider if these infringe the rights of people with dementia. This is not done in an accusatory manner but in a way designed to make sense of the care partnership. Knowledge of and respect for spatial boundaries encourages carers to connect with people with dementia first, before intruding into their personal or intimate space.

This forms part of the third aspect of PAC - connecting with the person with dementia. Acquiring permission to physically connect with the person who has dementia, rather than initiating contact without invitation, is the cornerstone of this aspect of the training.

PAC helps carers to understand how someone with dementia may feel if their personal or intimate space is infringed without warning and that reactive responses may be the inevitable result if carers are not sensitive in their approach. Part of this 'positive physical approach' is hand-under-hand ( $\mathrm{HUH})$, which further strengthens the connection between the carer and the person with dementia. HUH is a step up from the usual handshake - it helps carers to connect with people who have dementia while still give them a sense of control (Worcester 2014).

$\mathrm{HUH}$ is a technique in which the care partner has their hand under the hand of a person living with dementia. Their palms are touching, which offers a comforting and supportive connection. This allows for a natural eating movement and triggers muscle memory, giving the person living with dementia the feeling of control (Nolan 2016). It can help support nutrition in care facilities by enabling carers to assist with eating and drinking, and improving nutritional intake (Batchelor-Aselage et al 2015). Interactive exercises are used to generate an appreciation of techniques to offer supportive care.

PAC's theoretical origins, including person-centred care, are evident throughout its training and resources. What matters is how these theories are given context. The heavy emphasis on people with dementia and how best to support them ensures that the theories begin to make sense without the need to refer to them. The facts and theories are linked to promote a deeper understanding, such as how the anticipated visual changes affect the perception of people with dementia and thus how carers should physically contact them.

\section{References}

Alzheimer's Society (2014) Dementia UK. Second edition. Alzheimer's Society. London.

Alzheimer's Society (2016) Fix Dementia Care: Hospitals. Alzheimer's Society: London.

Batchelor-Aselage M, Amella EJ, Broome Rose S et al (2015) Dementia-related mealtime difficulties: assessment and management in the long-term care setting. In Bales CW, Locher JL, Saltzman E (Eds) Handbook of Clinical Nutrition and Aging. Third edition. Humana Press, New York NY.

Brooker D (2007) Person-Centred Dementia Care: Making Services Better. Jessica Kingsley Publishers, London.
Chater K, Hughes N (2013) Strategies to delive dementia training and education in the acute hospital setting. Journal of Research in Nursing $18,6,578-593$

Dementia UK (2013) Curriculum for UK Dementia Education. Dementia UK, London.

Department of Health, Social Services and Public Safety (DHSSPS) (2011) Improving Dementia Services in Northern Ireland: A Regional Strategy. DHSSPS, Belfast

Department of Health (2015) Prime Minister's Challenge on Dementia 2020. DH London.
Elvish R, Burrow S, Cawley R et al (2014) 'Getting to Know Me': the development and evaluation of a training programme for enhancing skills in the care of people with dementia in general hospita settings. Ageing and Mental Health. 18, 4, 481-488.

Elvish R, Burrow S, Cawley R et al (2016) 'Getting to Know Me': The Second Phase Roll-Out of a Staff Training Programme for Supporting People with Dementia in General Hospitals. dx.do org/10.1177/1471301216634926 (Last accessed: 30 November 2016.
Fox C, Maidment I, Moniz-Cook E (2013) Optimising primary care for people with dementia. Mental Health in Family Medicine. 10, 3, 143-151.

Health Education England (HEE) (2015) Dementia Core Skills Education and Training Framework. HEE, London.

Jones C, Moyle W, Stockwell-Smith G (2013) Caring for older people with dementia: an exploratory study of staff knowledge and perception of training in three Australian dementia care facilities. Australasian Journal on Ageing. 32, 1, 52-55. 
Links are made between the brain change and the capacity to understand and accept care, and how PAC can promote a successful care partnership. This approach combines theoretical and practical information with a focus on the practical, an aspect identified by students as important and valuable (Chater and Hughes 2013).

\section{Community and primary care nurses}

There is a suggestion that people with dementia occupy $25 \%$ of hospital beds (Alzheimer's Society 2016). Whatever the number, the remaining of the 850,000 people in the UK with dementia will be cared for in community and primary care settings. Fox et al (2013) and Wilcock et al (2013) emphasised that primary care is pivotal to the good assessment and care of people with dementia, as it is the first point of contact for most of them and for their caregivers.

PAC training could be invaluable for community and primary care nurses. It can help those who receive it to appreciate care as a partnership to understand what someone with dementia may be experiencing and how a receptive and considerate approach may ease any anxieties or uncertainties.

This knowledge and practical understanding can also be shared with informal carers, to support them in their role and help them to adapt their approach to meet the needs of the person with dementia.

\section{Conclusion}

Dementia training and education focuses on theoretical explanations of pathology, complicating factors, and challenges associated with care or the diseases causing the dementia. Community and primary care nurses can investigate many aspects of person-centred dementia information through internet search engines, social networking sites, blogs and video-sharing sites.

PAC is a different way of understanding dementia's pathologies. Most significantly, it gives practical advice on how to connect and interact with people with dementia - to 'do with' rather than 'do to'.

Sensitivity, compassion, understanding and flexibility are encouraged in PAC, enhancing and preserving the dignity of the person with dementia. Snow's mantra is that 'Dementia doesn't rob someone of their dignity - it's our reaction to them that does.' By promoting a positive approach to care, dignity is preserved and positive outcomes are experienced.

The opportunity to access PAC training is increasing, and there are many resources available that can inform nurses, including how it can improve their practice in dementia care and their ability to support informal carers.

The culture of care in relation to dementia is evolving. It is an ideal opportunity for community nurses to be at the forefront of this change in culture, to promote positive attitudes and new initiatives in dementia care.
Kolb D (1984) Experiential Learning: Experience as the Source of Learning and Development. Prentice Hall, Englewood Cliffs NJ.

Maddox MK, Burns T (1997) Positive approaches to dementia care in the home. Geriatrics. 52 , Suppl 2, S54-S58.

NHS Education Scotland (2011) Promoting Excellence: A Framework for All Health and Social Services Staff Working with People with Dementia, Their Families and Carers. The Scottish Government, Edinburgh
Nolan BAD (2016) Eating and Dementia: How Language Guides our Actions! Positive Approach to Care. Online Dementia Journal. http://files.ctctcdn. com/53c9860b201/5af54b8d-bfde-49db-9dla8289f70a24d6.pdf?utm_source=January+2016++Online+Dementia+Journal\&utm_campaign=2016 ODJ_January\&utm_medium=email (Last accessed: 12 December 2016.)

Nuffield Council on Bioethics (2009) Dementia Ethical Issues. Nuffield Council on Bioethics, London. O'Connor D, Purves B (2009) (Eds) Decision Making, Personhood and Dementia: Exploring the Interface. Jessica Kingsley Publishers, London.
Passalacqua SA, Harwood J (2012) VIPS communication skills training for paraprofessiona dementia caregivers: an intervention to increase person-centred dementia care. Clinica Gerontologist. 35, 5, 425-445.

Possin KL (2010) Visual spatial cognition in neurodegenerative disease. Neurocase. 16, 6 466-487.

Raymond M, Warner A, Davies N et al (2014) Evaluating educational initiatives to improve palliative care for people with dementia: a narrative review. Dementia. 13, 3, 366-381.
Royal College of Psychiatrists (RCP) (2011) Report of the National Audit of Dementia Care in Genera Hospitals 2011. RCP, London.

Snow T (2016a) Positive Approach to Brain

Change. www.teepasnow.com (Last accessed: 20 January 2017.)

Snow T (2016b) The GEMS ${ }^{\circ}$ : Brain Change Model teepasnow.com/events/teepas-gems/ (Last accessed: 12 December 2016. 